Juli 2021 | innsbruck university press, Innsbruck

ZDS | ISSN 2791-4313 | www.zds-online.org

Nr. 1/2021| DOI 10.15203/ZDS_2021_1.07 | internes Review

Lizenz: CC BY 4.0 (Namensnennung)

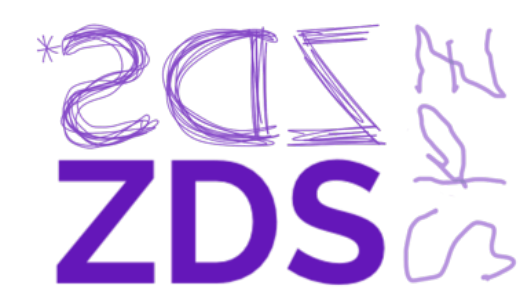

Michael Zander

\title{
Corona-Pandemie und Behinderung - ein Überblick
}

\section{Zusammenfassung}

Der Beitrag gibt einen Überblick über die gesellschaftliche Lage behinderter und chronisch kranker Menschen in der Pandemiekrise. Dargestellt und diskutiert werden unter anderem Befunde und Positionen zu den sozial-ökologischen Charakteristika der Pandemie, zu erhöhten Erkrankungs- und Sterberisiken in Institutionalisierung der Behindertenhilfe sowie zur Triage und dem Aufkommen sozialdarwinistisch getönter Verschwörungsideologien. Abschließend werden Fragen zur Krisenbewältigung aufgeworfen.

Schlüsselwörter: Behinderung, chronische Krankheit, Covid-19-Pandemie, Triage, Sozialdarwinismus

\section{Corona Pandemic and Disability - An Overview}

\section{Abstract in English}

This article provides an overview of the social situation of disabled and chronically ill persons in the pandemic. It discusses findings and positions on the social-ecological characteristics of the pandemic, the increased risks of illness and mortality in institutionalized disability care, as well as triage and the emergence of social Darwinist-tinged ideologies of conspiracy. Finally, questions about crisis management are raised.

Keywords: disability, chronic disease, Covid-19-pandemic, triage, social Darwinism 


\section{Einleitung}

Es ist, als wäre mit der globalen Coronavirus-Pandemie eine Dystopie wahr geworden. Der neuartige Erreger SARS-CoV-2 bedroht potenziell alle Menschen, insbesondere aber Angehörige sogenannter Risikogruppen, also ältere Menschen und Personen mit bestimmten chronischen Erkrankungen und Beeinträchtigungen. Dieser Umstand legt es unter anderem nahe, den gesellschaftlichen Umgang mit der Pandemie aus der Perspektive der Disability Studies zu untersuchen, zu deren zentralen Forschungsgegenständen die Ausgrenzung behinderter und chronisch kranker Menschen gehört (Zander, 2016).

Anfang Februar 2020 machte die Redaktion der Zeitschrift für Disability Studies ihrem Call for Papers für die erste Ausgabe bekannt. Damals war noch nicht abzusehen, wie aktuell das Thema "Utopie und Dystopie“ im Laufe der folgenden Monate werden sollte. Das Virus, das sich seit November 2019 von seinem vermuteten Ursprungsort in China allmählich über den Erdball verteilte, wurde vor allem in Europa und den Vereinigten Staaten zunächst unterschätzt. Erst ab Ende Februar 2020 traten in Deutschland, Österreich und der Schweiz die ersten Infektionen und dadurch ausgelöste Covid-19-Erkrankungen auf. Länder wie Italien, Spanien und Frankreich wurden früh und besonders schwer von der Pandemie getroffen. Dort gab es bereits im Mai 2020 landesweit jeweils um die 30.000 Tote und hunderttausende Infizierte.

Will man einen Überblick zum Thema Behinderung in Zeiten der Pandemie geben, stößt man zunächst auf einige methodische und theoretische Schwierigkeiten. Dies betrifft zunächst einmal den Behinderungsbegriff und damit die Frage, um wessen Lebenssituation es in der Darstellung gehen soll. Denkbar wäre, von einem Minderheiten- oder Randgruppenmodell auszugehen. Im Rahmen eines dementsprechend eher engen Behinderungsbegriffs wären vor allem Menschen gemeint, die mit einer schwerwiegenden Erkrankung oder Beeinträchtigung zur Welt gekommen sind oder sich diese in jungen oder mittleren Jahren zugezogen haben. Die Unterscheidung zwischen Behinderung, Psychiatrie und (pflegebedürftigem) Alter spiegelt sich nicht nur in den gesetzlich geregelten "Hilfesystemen“ wider, sondern manifestiert sich auch in verschiedenen sozialen Bewegungen (Krüppelbewegung, Psychiatriebetroffene, Graue Panther) sowie in spezifischen sozialwissenschaftlichen Ansätzen (Disability Studies, Mad Studies, Critical Gerontology). Die Unterscheidung hat durchaus ihre Berechtigung, erlaubt sie es doch, sich auf gemeinsame Lebensbedingungen und Erfahrungen zu beziehen, ungeachtet der Diversität innerhalb der jeweiligen Gruppe. Sie stößt in der Pandemie aber an Grenzen, da das Minderheitenmodell einen großen Teil der am stärksten Betroffenen nicht berücksichtigt, also vor allem Menschen mit altersassoziierten Beeinträchtigungen. Eine Alternative wäre, Beeinträchtigung weiter zu fassen, also eher als graduelles Phänomen aufzufassen, von dem sehr viele Menschen betroffen sind, etwa im Hinblick auf die Wahrscheinlichkeit für schwere Verläufe von CovidErkrankungen. Durch ein solches Verständnis bezieht man die vulnerabelsten Gruppen mit ein, darf aber gleichzeitig die Belange der Minderheit nicht außer Acht lassen. Die hier zu referierenden Befunde rekurrieren mal auf den engen, mal auf den weiten Behinderungsbegriff.

Ein weiteres Problem besteht in der Wahl des Darstellungsrahmens. Viele Untersuchungen konzentrieren sich auf die unmittelbaren und gruppenspezifischen Folgen der Pandemie und der Präventionsmaßnahmen. Dabei läuft man allerdings Gefahr, andere vulnerable Gruppen zu vernachlässigen und außerdem die tieferliegenden Ursachen der gegenwärtigen Krise zu übergehen. Man sollte also den Darstellungsrahmen so erweitern, dass die Komplexität des Geschehens deutlich wird. Angesichts der Pandemie ist es wichtig, die Lage behinderter Menschen nicht isoliert zu betrachten, sondern sie in größere gesellschaftliche und ökologische Zusammenhänge einzuordnen. Für eine angemessene Analyse ist es notwendig, zwischen der Faktizität der Pandemie einerseits und den politischen Handlungsoptionen sowie den sich daraus ergebenden erwünschten und unerwünschten Konsequenzen zu differenzieren. Da in der öffentlichen Diskussion diese Differenzierung häufig nicht gemacht wird, muss der aktuelle Forschungsstand zu Corona eingangs wenigstens kurz umrissen werden. 


\section{Eine globale Naturkatastrophe}

Die generelle Gefährlichkeit der Pandemie lässt sich ermessen an der Zahl der bereits verstorbenen Infizierten, an den Langzeitfolgen und an den Übertragungswegen des Virus. Nach Angaben des deutschen Robert-Koch-Instituts (RKI) ist der Anteil der Verstorbenen an den diagnostizierten Krankheitsfällen altersabhängig; er liegt bei Erkrankten bis etwa 50 Jahren bei unter 0,1\%, und steigt danach auf über 10\% bei den über 80-Jährigen an (RKI, 2021a). Sogenannte Vorerkrankungen wie Diabetes mellitus, Herzerkrankungen, Schlaganfall, chronische Nierenprobleme oder Adipositas erhöhen das Risiko für einen schweren Krankheitsverlauf. Einer Untersuchung aus dem Robert-Koch-Institut zufolge gehören in Deutschland 36,5 Millionen Menschen zur „Risikogruppe“ und davon 21,6 Millionen zur „Hochrisikogruppe“ (Rommel et al., 2021). Die meisten Betroffenen leben demnach in den ostdeutschen Bundesländern und im Saarland. Über die möglichen Langzeitfolgen einer Erkrankung („long covid“) wird in der medizinischen Fachliteratur diskutiert. Berichtet wird unter anderem von Müdigkeitserscheinungen und Gedächtnisproblemen, zu denen es auch nach milden Verläufen der eigentlichen Krankheit kommen kann (Mahase, 2020). Besonders gefährlich ist das SARS-CoV-2-Virus deshalb, weil es durch die Luft - in Form sogenannter Aerosole - übertragbar ist und weil Infektionen oft unerkannt bleiben, aber ihrerseits zu neuen Ansteckungen führen. Staatliche Verordnungen zur Prävention - darunter Abstandsregeln, Maskenpflicht, Kontaktbeschränkungen, Ausgangssperren - zielen vor allem darauf, ein exponentielles Wachstum der Infektionen und damit eine mögliche Überlastung der Gesundheitssysteme und anderer essentiell wichtiger („systemrelevanter") gesellschaftlicher Institutionen zu verhindern. Dabei sind die Konzentration auf den Privatbereich und die weitgehende Ausklammerung der industriellen und landwirtschaftlichen Produktion vom "Lockdown" umstritten, wobei unter anderem die Initiative ZeroCovid maßgeblich zur öffentlichen Diskussion beigetragen hat (https://zero-covid.org; Lee, Morris \& Sundborn, 2020).

Bei der Bekämpfung der Pandemie verfolgen die meisten Staaten ähnliche Strategien. Zu den Ausnahmen gehörten bisher die USA. Der im November 2020 bzw. im Januar 2021 abgewählte US-Präsident Donald Trump und die bis dahin regierende Republikanische Partei verharmlosten das Virus. Bis zum 23. Februar 2021 starben nach Angaben der Weltgesundheitsorganisation WHO weltweit fast zweieinhalb Millionen infizierte Menschen; die USA hatten fast eine halbe Million Todesopfer zu beklagen, in Deutschland waren es knapp 68.000, in Österreich rund 8.200, in der Schweiz 9.100 (WHO Dashboard, Stand 23.2.2021). Wie viele davon eine behördlich anerkannte Behinderung hatten, ist gegenwärtig nicht bekannt.

Der Ursprung des SARS-CoV-2-Virus ist bislang noch nicht abschließend geklärt. Es gilt als sehr wahrscheinlich, dass der Erreger 2019 von einem Wildtier - möglicherweise über einen Zwischenwirt - auf den Menschen überging (Wu et al., 2020). Die Pandemie als solche ist eine Naturkatastrophe, für deren Ausbruch unmittelbar niemand verantwortlich ist, ähnlich wie im Fall der sogenannten Spanischen Grippe zu Beginn des 20. Jahrhunderts. Allerdings weist die Forschung bereits seit längerem auf menschengemachte Bedingungen hin, die die Gefahr einer Zoonose, also das Überspringen von Erregern von Tieren auf den Menschen, erhöhen, vor allem das Artensterben und die Zerstörung natürlicher Habitate, durch die Kontakte zwischen Menschen und wild lebenden Tieren wahrscheinlicher werden (Johnson et al., 2020; Walzer, 2020). Wenn es richtig ist, dass ökonomisch-ökologische Prozesse die Wahrscheinlichkeit von Pandemien erhöhen und dass behinderte Menschen in besonderem Maße durch Pandemien gefährdet sind, dann sind jene ökonomisch-ökologischen Prozesse relevant für Disability Studies und Behindertenpolitik.

\section{Behinderung, Pandemie und Triage}

Die Folgen der Pandemie und der Präventionsmaßnahmen sind nicht nur ein naturwissenschaftliches, sondern auch ein genuin gesellschaftliches Problem. Sie sind komplex und momentan noch nicht zu überblicken. Es gibt Hinweise darauf, dass die Risiken, gesundheitlichen oder finanziellen Schaden zu erleiden, sozial ungleich verteilt sind. So ist die Wahrscheinlichkeit, an Covid-19 zu sterben, in ärmeren Gegenden Großbritanniens höher als in wohlhabenden, u.a. weil ärmere Menschen weniger Möglichkeiten haben, sich zu schützen, z.B. durch Homeoffice (Marmot \& Allen, 2020; zur sozialen Ungleichheit angesichts 
der Pandemie s.a. Zander, 2021). Knapp 30\% der in Deutschland Verstorbenen erhielt Leistungen eines Pflegedienstes oder lebte in Pflegeheimen und anderen Einrichtungen, einschließlich Asyl- und Obdachlosenunterkünften sowie Justizvollzugsanstalten (RKI, 2021b).

Behinderte Menschen, die in Einrichtungen leben, sind besonders gefährdet. Forderungen nach einer „emergency deinstitutionalisation“ (Goldman et al., 2020), einer Notfall-Ambulantisierung zum Zweck der Prävention und zur Wahrung der Menschenrechte behinderter Menschen, wie sie unter anderem das European Network for Independent Living erhebt (ENIL, 2020), wurden bisher nicht umgesetzt oder auch nur in einer größeren Öffentlichkeit diskutiert. Studien deuten darauf hin, dass Erkrankungsrisiken in den eigenen vier Wänden und in kleineren Wohneinheiten geringer sind als in größeren Einrichtungen der Behindertenhilfe (Landes et al., 2020; Landes et al., 2021; Perera et al., 2020). Erhöhte Erkrankungs- und Sterberisiken für Menschen mit "geistiger" Behinderung werden aus Schweden, den Niederlanden und den USA berichtet (Habermann-Horstmeier, 2021). Insbesondere stationäre Unterbringung, Einrichtungsgröße und Personalwechsel scheinen eine wichtige Rolle für vermehrte Krankheits- und Todesfälle zu spielen (Habermann-Horstmeier, 2021). In England starben nach einer Schätzung des britischen Office for National Statistics (ONS, 2021) zwischen dem 24. Januar und dem 20. November 2020 knapp 204.000 Infizierte im Alter von 30 bis 100 Jahren, die in ihrer Mehrheit bei einer Zensusbefragung von 2011 angegeben hatten, "less" oder "more-disabled" zu sein, d.h. hier wurden Selbsteinschätzungen und ein breiterer Behinderungsbegriff zugrunde gelegt. Abhängig von Schweregrad und Geschlecht lag demnach das Risiko für behinderte Menschen, im Zusammenhang mit einer SARS-CoV-2-Infektion zu sterben, zwischen zwei- bis dreieinhalbmal so hoch wie für nichtbehinderte Menschen der Altersgruppe (ONS, 2021).

Dass sich die Auswirkungen der Pandemie und ihrer Eindämmung auf das Leben der im engeren Sinne behinderten Menschen noch nicht genauer abschätzen lassen, liegt nicht zuletzt an fehlenden Daten (Kuper et al., 2020; Reed et al., 2020). In Deutschland erheben weder die Gesundheitsämter noch die Träger der Eingliederungshilfe systematisch Daten zu Krankheits- und Sterbefällen (Habermann-Horstmeier, 2021). Nach Einschätzung behindertenpolitischer Interessenvertretungen wurden der Schutz und die Belange behinderter Menschen, die in Einrichtungen oder in der eigenen Wohnung leben, zu spät und nicht hinreichend berücksichtigt (DBR, 2020; ISL, 2021). Für Menschen, die in der eigenen Wohnung leben und auf persönliche Assistenz angewiesen sind, insbesondere im Rahmen des sogenannten Arbeitgebermodells, fehlte es zunächst an Schutzausrüstungen und Schnelltests. Dieser Mangel wird mittlerweile mehr und mehr beseitigt, nicht zuletzt auf Initiative von Organisationen der Behindertenbewegung (in Berlin etwa die Arbeitsgemeinschaft für selbstbestimmtes Leben schwerstbehinderter Menschen, ASL).

Medienberichten zufolge kam es in Behinderteneinrichtungen immer wieder zu Corona-Ausbrüchen; ferner wurden Entgeltkürzungen in Behindertenwerkstätten mit einer pandemiebedingt einbrechenden Auftragslage begründet, ein Vorgehen, gegen das sich vereinzelt Widerstand rührt (Buhl, 2020; Grimmer, 2020; Kurbjeweit, 2020). Der Disability Rights Monitor, eine internationale Online-Befragung behinderter Menschen und ihrer Angehörigen, ermittelte Menschenrechtsverletzungen, etwa Gewalt, Freiheitsentzug oder eingeschränkten Zugang zu Gesundheitsversorgung, aber die Befunde sind wenig detailliert und nicht nach Ländern aufgeschlüsselt (DRM, 2020).

Für Konfliktstoff sorgen die Empfehlungen deutscher Fachgesellschaften für Intensivmedizin zur Triage, also zur Zuteilung knapper medizinischer Ressourcen in akuten Notfällen. Neun behinderte Menschen haben Verfassungsbeschwerde eingelegt, um den Gesetzgeber zu einer Regelung zu veranlassen. Das Forum behinderter Juristinnen und Juristen kritisiert, dass die bisherigen Empfehlungen verfassungswidrig seien, weil sie Komorbidität, Lebenszeiterwartung und Gebrechlichkeit bei der Entscheidung einbezögen und damit behinderte Menschen diskriminierten (FbJJ, 2020). Ähnliche Kontroversen gibt es auch in Österreich (ÖBR, 2020) und der Schweiz (Scheidegger, 2020). Umstritten ist insbesondere - auch international (Festen et al., 2021) - der Einsatz der Clinical Frailty Scale in Entscheidungsprozessen zur Triage, weil die Angewiesenheit auf personelle Unterstützung sowie auf Hilfsmittel wie Rollator und Rollstuhl den Punktwert erhöht, wodurch behinderte Menschen diskriminiert werden (FbJJ, 2020). Da die Abwägung von Leben gegen die Menschenrechte verstößt, werden für akute Notfälle, in denen die Rationierung lebenswichtiger medizinischer Mittel notwendig ist, folgende Alternativen vorgeschlagen: „das Prioritätsprinzip (wer war 
zuerst da?), das Dringlichkeitsprinzip (wer braucht die Behandlung am notwendigsten?)“ oder "das Zufallsprinzip“ (BODYS, 2020, S. 5).

In Spanien läuft ein Gerichtsprozess gegen die Chefin der konservativ-rechtspopulistischen Regionalregierung von Madrid; sie soll illegale Triage-Maßnahmen angeordnet haben (Wandler, 2020). Unter Verweis auf Zahlen des deutschen RKI hat die Grünen-Politikerin Corinna Rüffer darauf aufmerksam gemacht, dass rund zwei Drittel der Todesopfer in Deutschland nicht auf einer Intensivstation sterben, sondern u.a. im Pflegeheim. Sie äußerte den Verdacht, dass dies auch auf eine "versteckte Triage" zurückzuführen sei, d.h. darauf, dass Pflegebedürftige nicht rechtzeitig ins Krankenhaus gebracht werden (Szent-Ivanyi, 2021). Die Entstehung von akuten Notsituationen, in denen medizinische Versorgungsleistungen mit potenziell tödlichen Folgen rationiert und zugeteilt werden müssen, wird nicht zuletzt begünstigt und provoziert durch eine Gesundheitspolitik, die auf Finanzkürzungen und -beschränkungen ausgerichtet ist (Horton, 2021).

\section{Sozialdarwinismus und Verschwörungsideologien}

Vor allem zu Beginn der Pandemie meldeten sich in deutschen Medien Stimmen zu Wort, die das Leben alter und pflegebedürftiger Menschen mehr oder weniger unverhohlen zur Disposition stellten. So forderten Bettina Schöne-Seifert, ehemaliges Mitglied des Deutschen Ethikrats, und Hugo van Aken (Aken \& SchöneSeifert, 2020), es müsse „für jeden einzelnen Heimbewohner" geklärt werden, „ob er im hypothetischen Fall einer schweren Covid-19-Erkrankung (...) intensivmedizinisch behandelt werden“ wolle. Der "Tod an einer Lungenentzündung" müsse "nicht in jedem Einzelfall bekämpft" werden. Der Autorin und dem Autor unterläuft offensichtlich ein Lapsus, wenn sie schreiben, die „Herausforderung" einer „Durchseuchung vieler Seniorenheime“ erhalte zu wenig „Unterstützung“ (Aken \& Schöne-Seifert, 2020.). Der Tübinger Oberbürgermeister Boris Palmer (Bündnis 90/Die Grünen) plädierte für eine schnelle Beendigung des ,Lockdowns“ und behauptete: „Ich sage es Ihnen mal ganz brutal: Wir retten in Deutschland möglicherweise Menschen, die in einem halben Jahr sowieso tot wären" (zit. n. N.N., 2020). Der Finanzinvestor Alexander Dibelius fragte suggestiv, ob es richtig sei, dass „zehn Prozent der (...) Bevölkerung geschont (...), 90 Prozent samt der gesamten Volkswirtschaft aber extrem behindert“" würden (zit. n. Tuma, 2020).

Neben derartigen offen abwertenden Stellungnahmen, die das Lebensrecht alter, kranker und behinderter Menschen mehr oder weniger infrage stellen (Grams, 2021), gibt es Positionen, die zwar subtiler formuliert sind, inhaltlich aber in eine ähnliche Richtung gehen und menschliches Leben recht kaltschnäuzig einem ökonomischen Nutzenkalkül unterwerfen. Dies gilt etwa für ein Papier aus dem „arbeitgebernahen“ Institut der deutschen Wirtschaft (IW). Die Autoren rechnen polemisch-populistisch mit der Pandemiebekämpfung der Bundesregierung ab und fordern, es müsse ökonomisch abgewogen werden, „wie viele Corona-Fälle und auch Corona-Tote in einer Welt mit umfassend verfügbarem Impfstoff hinnehmbar" seien; künftige Infektionswellen seien "durch Impfung so einzudämmen, sodass es (...) weiterer Maßnahmen nicht bedarf“ (Bardt \& Hüther, 2021, S. 10).

Seit dem Sommer 2020 kommt es in verschiedenen Ländern immer wieder zu Protestdemonstrationen gegen die Eindämmungsmaßnahmen der Regierungen. Die meisten Teilnehmenden vertreten Verschwörungsideologien, nach denen die Pandemie von der Regierung absichtlich herbeiführt worden sei oder in ihrer Gefährlichkeit übertrieben werde (Zander, 2020). „Sacrifice the weak" - „opfert die Schwachen“ - ist eine besonders drastische Parole, die auf dem Schild einer Anti-Lockdown-Demonstrantin und TrumpAnhängerin in Tennessee, USA, zu lesen war (Kindred, 2020). Andere verbinden ihren Protest gegen die Präventionsmaßnahmen mit der Forderung, „Risikogruppen“ zu „schützen“. Damit verbinden sie die Vorstellung, dass Covid-19 nur für Angehörige der „Risikogruppen“ ernsthafte Folgen habe und diese Menschen von der übrigen Gesellschaft isoliert werden könnten - beides ist falsch, letzteres auch nicht wünschenswert. 
Politisch sind die Proteste in Deutschland und anderen Ländern stark von rechtspopulistischen und neofaschistischen Kräften geprägt (Aschenbrenner, 2020; Nachtwey, Schäfer, \& Frei, 2020). In geringerem Maße leugnen auch Menschen die Realität der Pandemie, die sich selbst als politisch links bezeichnen. Ein prominentes Beispiel ist der postmoderne italienische Philosoph Giorgio Agamben (2020), der in der Pandemie eine „Erfindung" des Staates sieht.

\section{Zukunftsfragen}

Sollte es im Laufe des Jahres 2021, v.a. mit Hilfe von Impfstoffen, gelingen, die Pandemie nachhaltig einzudämmen und damit Verschwörungsideologien zumindest teilweise den Boden zu entziehen, werden sich neue politische Aufgaben stellen. Angesichts vieler offener Fragen braucht es weitere Aufklärung darüber, welche Bevölkerungsgruppen in welcher Weise von den Folgen der Pandemie getroffen wurden. Insbesondere ist eine politische und gegebenenfalls auch eine juristische Aufarbeitung staatlicher und gesellschaftlicher Versäumnisse notwendig, die lebensbedrohliche Konsequenzen hatten. In Großbritannien wurde bereits eine „People's Covid Inquiry“ (Horton, 2021) ins Leben gerufen, die für ähnliche Initiativen in anderen Ländern ein Vorbild sein könnte.

Damit sich Fehler im Umgang mit der Pandemie künftig nicht wiederholen, ist es geboten, die politischen Selbstvertretungsorganisationen behinderter, alter und psychiatriebetroffener Menschen systematisch in die Planungen zur Bekämpfung von Pandemien und der Folgen anderer Naturkatastrophen einzubeziehen. Allerdings muss man sich klar machen, dass eine Krise wie die Corona-Pandemie durch massive Dilemmata und Interessenkonflikte gekennzeichnet ist, deren Entscheidung in starkem Maße von politischen Mehrheiten und gesellschaftlichen Kräfteverhältnissen abhängt. (Man denke etwa an die Preisgestaltung bei Impfstoffen und die politisch und ökonomisch bedingte globale Impfungerechtigkeit.)

In ihren Empfehlungen für eine „inklusive Antwort“ auf die Pandemie empfehlen die Vereinten Nationen, die Schutzmaßnahmen in stationären Einrichtungen zu verbessern und darüber hinaus die Zahl der in Institutionen lebenden Menschen zu reduzieren. Es brauche zum einen verbindliche Strategien, um den Prozess der Deinstitutionalisierung zu beschleunigen, zum anderen eine Ausweitung gemeindenaher Unterstützungssysteme (United Nations, 2020). Es muss allerdings hinzugefügt werden, dass Wohnformen vor allem bedarfsgerecht sein müssen, unabhängig davon, ob es sich um größere Einrichtungen, Wohngemeinschaften oder Wohnen in der Gemeinde handelt. Probleme wie Ressourcen- und Personalmangel, schlechte Bezahlung von Mitarbeitenden, Einsamkeit, Vernachlässigung oder Gewalt können im Rahmen verschiedener Wohnformen vorkommen.

Nach der Pandemie wird es nicht zuletzt notwendig sein, die finanziellen Folgen zu bewältigen. Den Spielraum dafür gäbe es, wie z.B. eine Studie der Hilfsorganisation Oxfam Deutschland (2021, S. 4) zeigt: „Das Vermögen der (im Dezember 2020) zehn reichsten Männer der Welt ist seit Februar 2019 - trotz der Pandemie - um fast eine halbe Billion US-Dollar auf 1,12 Billionen US-Dollar gestiegen. Dieser Gewinn wäre mehr als ausreichend, um die ganze Weltbevölkerung gegen Covid-19 zu impfen und sicherzustellen, dass niemand durch die Pandemie in die Armut gestürzt wird."

Es steht zu befürchten, dass in Zukunft - unter Verweis auf Staatsausgaben während der Pandemie öffentliche Sozial-, Gesundheits- und Teilhabeleistungen stark eingeschränkt werden. Außerdem könnten Regierungen die Belastung der öffentlichen Haushalte als Vorwand dafür nutzen, um gesellschaftliche Veränderungen zu blockieren. Notwendig ist insbesondere die weitere Inklusion behinderter Menschen sowie ferner eine Politik, die der Bedrohung durch Pandemien und durch die voranschreitende globale Erwärmung entgegenwirkt (zu Parallelen zwischen dem Kampf gegen die Covid-Pandemie und jenem gegen den Klimawandel siehe Klenert et al., 2020). Auf absehbare Zeit geht es um nichts Geringeres als darum, dass Eintreten weiterer Dystopien zu vermeiden. 


\section{Literatur}

Agamben, G. (2020, 26. Februar). The Invention of an Epidemic. European Journal of Psychoanalysis. https://www.journal-psychoanalysis.eu/coronavirus-and-philosophers/

Aken, H. v., \& Schöne-Seifert, B. (2020, 14. April). Corona und Medizinethik: Worauf es jetzt ankommt. Frankfurter Allgemeine Zeitung. https://www.faz.net/aktuell/feuilleton/debatten/einelungenentzuendung-in-hohem-alter-muss-nicht-immer-bekaempft-werden-16723313.html

Aschenbrenner, S. (2020, 12. November). Querdenken: Protestforscher Dieter Rucht über die Proteste. „Das Selbstbewusstsein der Demonstrierenden wächst". Jetzt.de. https://www.jetzt.de/politik/querdenkenprotestforscher-dieter-rucht-ueber-die-proteste?utm_source=pocket-newtab-global-de-DE

Bardt, H., \& Hüther, M. (2021). Aus dem Lockdown ins neue Normal. Institut der deutschen Wirtschaft. https://www.iwkoeln.de/fileadmin/user_upload/Studien/policy_papers/PDF/2021/IW-PolicyPaper_2021_Aus_dem_Lockdown_ins_neue_Normal.pdf

Buhl, J. (2020, 21. September). Wegen Corona: Lohnkürzungen in Behindertenwerkstätten. Job Inklusive. https://jobinklusive.org/2020/09/21/wegen-corona-pandemie-lohnkuerzungen-inbehindertenwerkstaetten/

BODYS, Bochumer Zentrum für Disability Studies. (2020, 14. April). Inklusion in Zeiten der KatastrophenMedizin: BODYS-Stellungnahme zur gegenwärtigen Triage-Debatte, in der behinderte Menschen hinten angestellt werden sollen. https://www.bodyswissen.de/files/bodys_wissen/Downloads/Stellungnahmen\%20und\%20Vortraege/BODYS_Stellungnah me_Inklusion\%20in\%20Zeiten\%20von\%20Katastrophenmedizin.pdf

DBR, Deutscher Behindertenrat. (2020, 25. September). Gleiche Teilhabe sicherstellen! Die Corona-Krise aus Sicht von Menschen mit Behinderung: Analysen und Forderungen. https://www.vdk.de/deutscherbehindertenrat/mime/00120852D1601564459.pdf

DRM, Disability Rights Monitor. (2020). Disability rights during the pandemic. A global report on findings of the Covid-19 Disability Rights Monitor. https://covid-drm.org/assets/documents/Disability-RightsDuring-the-Pandemic-report-web.pdf

ENIL, European Network on Independent Living. (2020, 30. Juni). Emergency Deinstitutionalisation: A joint call to act now! https://enil.eu/news/emergency-deinstitutionalisation-a-joint-call-to-act-now/

FbJJ, Forum behinderter Juristinnen und Juristen. (2020). Stellungnahme zu den Empfehlungen der Fachverbände für den Fall einer Triage. https://abilitywatch.de/wp-content/uploads/2020/04/FbJJStellungnahme-Triage-2020.pdf

Festen, D. A. M., Schoufour, J. D., Hilgenkamp, T. I. M., \& Oppewal, A. (2021). Determining Frailty in People with Intellectual Disabilities in the COVID-19 Pandemic. Journal of Policy and Practice in Intellectual Disabilities. https://doi.org/10.1111/jppi.12371

Goldman, P. S., van ljzendoorn, M. H., \& Sonuga-Barke, E. J. S. (2020). The implications of COVID-19 for the care of children living in residential institutions. The Lancet Child \& Adolescent Health, 4(6), e12. https://doi.org/10.1016/S2352-4642(20)30130-9

Grams, F. (2021). Corona wirkt wie ein Brennglas: Teilhabe und Ausgrenzung von Menschen mit Behinderungen. Forum Wissenschaft, 1, 49-52. 
Grimmer, C. (2020, 15. Oktober). Streit um Lohnkosten in Werkstätten für behinderte Menschen. Bayerischer Rundfunk. https://www.br.de/nachrichten/bayern/streit-um-lohnkosten-in-werkstaettenfuer-behinderte-menschen,SDE6oF2

Habermann-Horstmeier, L. (2021). Menschen mit geistiger Behinderung in Zeiten der COVID-19-Pandemie. Public Health Forum, 29(1), 64-67. https://doi.org/10.1515/pubhef-2020-0123

Horton, R. (2021). Offline: It's time to ask questions and learn lessons. Lancet, 397(10277), 865. https://doi.org/10.1016/S0140-6736(21)00571-7

ISL, Interessengemeinschaft Selbstbestimmt Leben. (2021, 16. März). Ein Jahr Corona: Ein Jahr vergessen und ungehört! Menschen mit Behinderungen ziehen Bilanz [Pressemitteilung]. https://islev.de/attachments/article/2474/PM\%20Ein\%20Jahr\%20Corona.\%20Ein\%20Jahr\%20vergessen\%20un d\%20ungeh\%C3\%B6rt!\%20Menschen\%20mit\%20Behinderungen\%20ziehen\%20Bilanz.pdf

Johnson, C. K., Hitchens, P. L., Pandit, P. S., Rushmore, J., Evans, T. S., Young, C. C. W., \& Doyle, M. M. (2020). Global shifts in mammalian population trends reveal key predictors of virus spillover risk. Proceedings of the Royal Society B: Biological Sciences, 287(1924), 20192736. http://dx.doi.org/10.1098/rspb.2019.2736

Kindred, A. (2020, 23. April). Anti-lockdown protester wields vile 'sacrifice the weak' poster at ReOpen Tennessee rally. The U.S. Sun. https://www.the-sun.com/news/724137/anti-lockdown-protestertennessee-rally/

Klenert, D., Funke, F., Mattauch, L., \& O'Callaghan, B. (2020). Five lessons from COVID-19 for advancing climate change mitigation. Environmental \& Resource Economics, 76(4), 751-778. https://doi.org/10.1007/s10640-020-00453-w

Kuper, H., Banks, L. M., Bright, T., Davey, C., \& Shakespeare, T. (2020). Disability-inclusive COVID-19 response: What it is, why it is important and what we can learn from the United Kingdom's response. Wellcome Open Research, 5, 79. https://doi.org/10.12688/wellcomeopenres.15833.1

Kurbjeweit, F. (2020, 2. September). Wilder Streik gegen Entgeltkürzungen. junge Welt.

Landes, S. D., Turk, M. A., Formica, M. K., McDonald, K. E., \& Stevens, J. D. (2020). Covid-19 outcomes among people with intellectual and developmental disability living in residential group homes in New York State. Disability and Health Journal, 13(4), 100969. https://doi.org/10.1016/j.dhjo.2020.100969

Landes, S. D., Turk, M. A., \& Wong, A. W. W.A. (2021). Covid-19 outcomes among people with intellectual and developmental disability in California: The importance of type of residence and skilled nursing care needs. Disability and Health Journal, 14(2),101051. https://doi.org/10.1016/j.dhjo.2020.101051

Lee, A., Thornley, S., Morris, A. J., \& Sundborn, G. (2020). Should countries aim for elimination in the Covid19 pandemic? BMJ, 370, m3410. https://doi.org/10.1136/bmj.m3410

Marmot, M., \& Allen, J. (2020). Covid-19: Exposing and amplifying inequalities. Journal of Epidemiology and Community Health, 74(9), 681-682. http://dx.doi.org/10.1136/jech-2020-214720

Mahase, E. (2020). Covid-19: What do we know about "long covid"? BMJ, m2815. https://doi.org/10.1136/bmj.m2815

Nachtwey, O., Schäfer, R., \& Frei, N. (2020). Politische Soziologie der Corona-Proteste: Grundauswertung. https://edoc.unibas.ch/80835/1/20210118133822_6005813e51e0a.pdf 
N.N. (2020, 28. April). Boris Palmer provoziert in Coronavirus-Krise: „Wir retten möglicherweise Menschen, die in einem halben Jahr sowieso tot wären." Der Tagesspiegel.

https://www.tagesspiegel.de/politik/boris-palmer-provoziert-in-coronavirus-krise-wir-rettenmoeglicherweise-menschen-die-in-einem-halben-jahr-sowieso-tot-waeren/25782926.html

ÖBR, Österreichischer Behindertenrat. (2020, 16. November). Triage: Menschen mit Behinderungen mehrfach gefährdet [Pressemitteilung]. https://www. behindertenrat.at/2020/11/triage-menschenmit-behinderungen-mehrfach-gefaehrdet/

ONS, Office for National Statistics. (2021). Updated estimates of coronavirus (COVID-19) related deaths by disability status, England: 24 January to 20 November 2020.

https://www.ons.gov.uk/peoplepopulationandcommunity/birthsdeathsandmarriages/deaths/articles/ coronaviruscovid19relateddeathsbydisabilitystatusenglandandwales/24januaryto20november2020

Oxfam Deutschland. (2021). Das Ungleichheitsvirus. Wie die Corona-Pandemie soziale Ungleichheit verschärft und warum wir unsere Wirtschaft gerechter gestalten müssen.

https://www.oxfam.de/system/files/documents/oxfam_factsheet_ungleichheitsvirus_deutsch.pdf

Perera, B., Laugharne, R., Henley, W., Zabel, A., Lamb, K., Branford, D., Courtanay, K., Alexander, R., Purandare, K., Wijeratne, A., Radhakrishnan, V., McNamara, E., Daureeawoo, Y., Sawhney, I., Scheepers, M., Taylor, G., \& Shankar, R. (2020). Covid-19 deaths in people with intellectual disability in the UK and Ireland: Descriptive study. BJPsych Open, 6(6), e123.

https://doi.org/10.1192/bjo.2020.102

Reed, N. S., Meeks, L. M., \& Swenor, B. K. (2020). Disability and Covid-19: Who counts depends on who is counted. The Lancet Public Health, 5(8), e423. https://doi.org/10.1016/S2468-2667(20)30161-4

RKI, Robert-Koch-Institut. (2021a, 19. April). Epidemiologischer Steckbrief zu SARS-CoV-2 und Covid-19. https://www.rki.de/DE/Content/InfAZ/N/Neuartiges_Coronavirus/Steckbrief.html

RKI, Robert-Koch-Institut. (2021b, 22. Februar). Täglicher Lagebericht des RKI zur Coronavirus-Krankheit2019 (Covid-19).

https://www.rki.de/DE/Content/InfAZ/N/Neuartiges_Coronavirus/Situationsberichte/Feb_2021/202102-22-de.pdf?_blob=publicationFile

Rommel, A., Treskova-Schwarzbach, M., Scholz, S., \& Lippe, E. von der (2021). Bevölkerung mit einem erhöhten Risiko für schwere COVID-19-Verläufe in Deutschland. Auswertungen der Studie GEDA 2019/2020-EHIS. Journal of Health Monitoring, 6(S2), 1-15. https://doi.org/10.25646/7858.3

Scheidegger, D. (2020, 4. November). Nach Kritik: Neue Triage-Richtlinien für ältere Covid-Patienten. Schweizer Radio und Fernsehen. https://www.srf.ch/news/schweiz/praezisierungen-zu-triagen-nachkritik-neue-triage-richtlinien-fuer-aeltere-covid-patienten

Szent-Ivanyi, T. (2021, 23. Januar). Corona: Grünen-Politikerin vermutet massenhaft versteckte Triage. Redaktionsnetzwerk Deutschland. https://www.rnd.de/politik/corona-grunen-politikerin-vermutetmassenhaft-versteckte-triage-SQ2X4YXT6VFH5K6SXZ6DR3QJMY.html

Tuma, T. (2020, 23. März). Investor Dibelius: „Shutdown der Wirtschaft macht mir mehr Angst als das Virus." Handelsblatt. https://www.handelsblatt.com/finanzen/anlagestrategie/trends/interviewinvestor-dibelius-shutdown-der-wirtschaft-macht-mir-mehr-angst-als-dasvirus/25671192.html?ticket=ST-9248465-gjfvLedwsbrUpcDd4g7b-ap1

United Nations. (2020). Policy Brief: A disability-inclusive response to Covid-19: May, 2020. http://unsdg.un.org/sites/default/files/2020-05/Policy-Brief-A-Disability-Inclusive-Response-toCOVID-19.pdf 
Walzer, C. (2020, 3. Dezember). Wenn Schnittstellen zu Wunden werden. Neue Zürcher Zeitung. https://www.nzz.ch/meinung/mensch-tier-pandemie-wenn-schnittstellen-zu-wunden-werdenld.1588569

Wandler, R. (2020, 6. Juni). Corona-Pandemie in Spanien: Verdacht auf Triage in Altersheimen. taz. https://taz.de/Corona-Pandemie-in-Spanien/!5690806/

Wu, D., Wu, T., Liu, Q., \& Yang, Z. (2020). The SARS-CoV-2 outbreak: What we know. International Journal of Infectious Diseases, 94, 44-48. https://doi.org/10.1016/j.ijid.2020.03.004

Zander, M. (2016). Disability Studies: Gesellschaftliche Ausgrenzung als Forschungsgegenstand. Bundesgesundheitsblatt, Gesundheitsforschung, Gesundheitsschutz, 59(9), 1048-1052. https://doi.org/10.1007/s00103-016-2394-8

Zander, M. (2020). Krise und Realitätsverlust. Sozialpsychologische Aspekte von Verschwörungsideologien. Forum Wissenschaft, 4, 9-12.

Zander, M. (i.E./ 2021). Psychosoziale Belastungen und ökonomische Ungleichheit in Zeiten der Pandemie. Kurswechsel, 1, 31-40.

\section{Autor_in}

Dr. Michael Zander vertritt derzeit die Professur "System der Rehabilitation“ im Studiengang Rehabilitationspsychologie an der Hochschule Magdeburg-Stendal. Seine Arbeitsschwerpunkte sind Disability Studies, Kritische Psychologie und Praxisforschung.

E-Mail: michael.zander@h2.de 